\title{
Advances in Wireless Communications and Networks
}

\author{
Hsiao-Hwa Chen • Chonggang Wang
}

Published online: 3 May 2011

(C) Springer Science+Business Media, LLC 2011

This special issue features five selected papers with high quality from the conference Chinacom 2009, which was held in Xi'An, China, August 26-28, 2009. This first article, "DFMAC: DTN-Friendly Medium Access Control for Wireless Local Area Networks Supporting Voice/Data Services", co-authored by Hao Liang and Weihua Zhuang, considers a heterogeneous network where local nodes reside in densely populated hot spots while nomadic nodes roam in a large area. Wireless local area networks (WLANs) are deployed in the hot spots, while a delay/ disruption tolerant network (DTN) provides services to nomadic nodes. In order to achieve efficient resource allocation for the DTN/WLAN integrated network, a DTN-friendly medium access control (DFMAC) scheme is proposed for the hot spots supporting voice/data services. Based on a superframe structure and token passing mechanisms, deterministic packet delay can be guaranteed for the voice services of local nodes, and a good tradeoff can be achieved for the data services between nomadic nodes and local nodes. Analytical and simulation results are

C. Wang $(\bowtie)$

InterDigital Communications,

King of Prussia,

Prussia, PA 19406, USA

e-mail: cgwang@ieee.org

H.-H. Chen

Department of Engineering Science,

National Cheng Kung University,

1 Da-Hsueh Road,

Tainan City 70101, Taiwan presented to demonstrate the performance of the proposed DFMAC scheme.

The second article titled "Sidelobe Suppression Using Extended Active Interference Cancellation with SelfInterferences Constraint for Cognitive OFDM Systems" from Zhiqiang Wang, Daiming Qu, Tao Jiang and Mahmoud Daneshmand, takes the theoretical analysis of the interfence cancellation to the next level, showing that a good sidelobe suppression performance of about $38.0 \mathrm{~dB}$ with signal-to-noise ratio loss less than $0.10 \mathrm{~dB}$ at symbolerror-rate of $10^{-3}$ when 64QAM modulation is employed, a critical asepct of cognitive OFDM systems, mathematically characterizing these features of prototypical OFDM waveforms.

The next article investigates Attribute-Based Encryption (ABE). The authors (Jin Li, Qian Wang, Cong Wang and Kui Ren) proposed a new technique on how to improve the efficiency of Attribute-ABE by utilizing the oftenfoundhierarchy relationships among the attributes that are inherent in many access control scenarios. As the first research effort along this direction, we coined the notion of hierarchical ABE (HABE), which can be viewed as the generalization of traditional $\mathrm{ABE}$ in the sense that both definitions are equal when all attributes are independent. We further gave a concrete HABE construction considering a tree hierarchy among the attributes. The construction can be proven secure under standard assumption. More importantly, our construction can exhibit significant improvement over the traditional $\mathrm{ABE}$ when attribute hierarchies exist.

The fourth article, "Joint optimization of source power allocation and relay beamforming in multiuser cooperative 
wireless networks," from Xin Li, Yimin D. Zhang and Moeness G. Amin, discusses the maximization of sum capacity in multiuser cooperative wireless networks consisting of single-antenna nodes, in which multiple concurrent source-destination links are relayed by a number of cooperative nodes. It is demonstrated that joint optimization of source power allocation and relay beamforming is important to enable significant sum capacity increment over TDMA, source selection, and equal-power spatial multiplexing schemes. The optimization of global sum capacity subject to individual capacity requirements is also considered to meet the needs of delivering data from each source node.

In this last article with the title "A-ADHOC: An Adaptive Real-time Distributed MAC Protocol for Vehicular Ad Hoc Networks", the authors (Jia Liu, Fengyuan Ren, Limin Miao and Chuang Lin) proposed an adaptive MAC protocol for Vehicular Ad Hoc Networks, referred to as AADHOC. The authored first pointed out that two major shortcomings of an existing MAC protocol, ADHOC, are its efficiency and reliability due to its fixed length of frames. A-ADHOC, with the adaptability of frame length, has improved the ADHOC in both channel resource utilization and response time. Through carefully devised frame adjusting rules, A-ADHOC not only lowers the response time by $50 \%$ over the original ADHOC protocol but improves the throughput greatly of the whole networks. Moreover, implementing mechanism of A-ADHOC substantially alleviates network failure when increasing dense of nodes, which is an inherent problem of ADHOC protocol.

The guest editors are thankful to our reviewers for their effort in reviewing the manuscripts. We also thank the Editin-Chief, Dr. Imrich Chlamtac for his supportive guidance during the entire process.

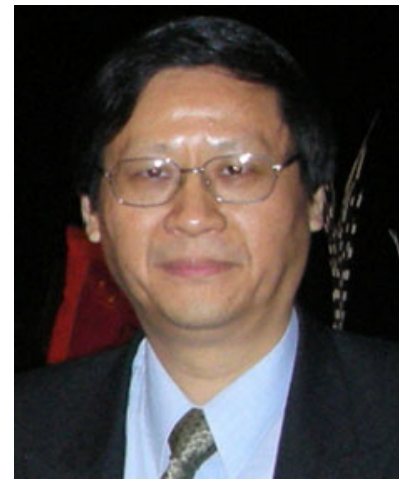

Dr. Hsiao-Hwa Chen $\left(\mathrm{S}^{\prime} 89-\mathrm{M}^{\prime}\right.$ 91-SM'00-F'10) is currently a Distinguished Professor in the Department of Engineering Science, National Cheng Kung University, Taiwan. He obtained his BSc and MSc degrees from Zhejiang University, China, and a $\mathrm{PhD}$ degree from the University of Oulu, Finland, in 1982, 1985 and 1991, respectively. He has authored or co-authored over 400 technical papers in major international journals and conferences, six books and more than ten book chapters in the areas of communications. He served as the general chair, TPC chair and symposium chair for many international conferences. He served or is serving as an Editor or/and Guest Editor for numerous technical journals. He is the founding Editorin-Chief of Wiley's Security and Communication Networks Journal (www.interscience.wiley.com/journal/security). He is the recipient of the best paper award in IEEE WCNC 2008 and a recipient of IEEE Radio Communications Committee Outstanding Service Award in 2008. He is a Fellow of IEEE, a Fellow of IET, and a Fellow of BCS

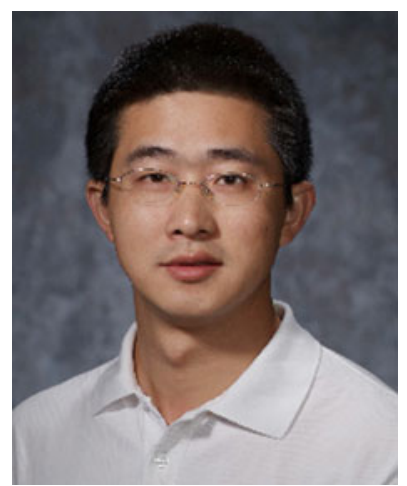

Dr. Chonggang Wang is a senior staff engineer in InterDigital Communications. His research interests include future Internet, machine-tomachine (M2M) communications, and wireless networks. He has published more than 80 journal/ conference articles and book chapters. He is on the editorial board for IEEE Communications Magazine, IEEE Networks Magazine, ACM/ Springer Wireless Networks, and Wiley's Security and Communication Networks Journal. He has served numerous IEEE conferences including ICNP, INFOCOM, Globecom, ICC and WCNC. He received his $\mathrm{Ph} . \mathrm{D}$ in computer science from Beijing University of Posts and Telecommunications in 2002. 